\title{
OTRI 3 - Detection of Mycoplasma sp. and Acholeplasma laidlawii in biological products and culture media by real time PCR
}

Natália Pedra Gonçalves ${ }^{1 *}$; Pedro Augusto Alves ${ }^{1}$; Érica Louro da Fonseca ${ }^{1}$; Darcy Akemi Okama ${ }^{1}$; Jaline Coutinho Silvério ${ }^{1}$; Daniel da Silva Guedes Júnior ${ }^{1}$.

\section{1 - Bio-Manguinhos/ Fiocruz}

\section{Introduction:}

Mycoplasma is the smallest less-wall prokaryotic organism and can that cause pneumonia and urogenital diseases in humans. Moreover, they frequently contaminate research and commercial laboratories involved in development and production of biological and pharmaceutical cell-derived products, enabling unreliable experimental results and unsafe biological products. The detection of Mycoplasma sp. and Acholeplasma laidlawii in biological products is a quality control test required by the European Pharmacopoeia (Eur. Ph.) and carried out by industries.

\section{Objective:}

To develop an in house real time PCR (qPCR) protocol for assessing $M y$ coplasma sp. and A. laidlawii in biological products and culture media for pharmaceutic industry.

\section{Methodology:}

The genome of eight species required by Eur. Ph. were selected in GenBank ${ }^{\circledast}$ for the $16 \mathrm{~S}$ ribossomal locus and aligned using the online tool ClustalOmega in order to select a common region for primer design. To achieve the specificity criteria, were excluded the regions shared with other phylogenetic related bacteria as Streptococcus, lostridium and Lactobacillus' genera. The primer design was analyzed through the online tool IDT OligoAnalyzer ${ }^{\oplus} 3.1$, and the PrepSEQ ${ }^{\circledR}$ kit was used for DNA extraction of ATCC pure cultures and biological products spiked with Mycoplasma and Streptococcus bovis, Clostridium sporogenes and Lactobacillus acidophilus, that are phylogenetically related to Mycoplasma sp. and A. laidlawii. The specificity and detection limit assays were made using $\mathrm{SYBR}^{\oplus}$ Green dye and the results were compared with the commercial kit for Mycoplasma detection MycoSEQ ${ }^{\mathrm{TM}}$ and convencional PCR. 


\section{Results:}

Four pairs of primers were designed and BLAST research revealed homology to 40 Mycoplasma species, including the species required by Eur. Ph. with $100 \%$ of identity. The qPCR tests using in house protocol demonstrated that the primers are specific for Mycoplasma genus and A. laidlawii, and the detection limit was less than $1 \mathrm{UFC} / \mathrm{mL}$ using pure culture of Mycoplasma. These results were similar to the obtained with the $\mathrm{MycoSEQ}^{\mathrm{rm}}$ and more sensitive than convencional PCR. Preliminary qPCR tests with biological products spiked with Mycoplasma has shown that in house protocol are also suitable and specific for this bacterial detection.

\section{Conclusion:}

The in house protocol for detection of Mycoplasma sp. and A. laidlawii by qPCR has shown to be as specific as the commercial kit MycoSEQ ${ }^{\mathrm{im}}$, with the ability to detect less than $1 \mathrm{UFC} / \mathrm{mL}$ of Mycoplasma. This represents lower costs and an alternative to the convencional PCR already established at the Quality Control Department - BioManguinhos, since qPCR presents advantages as higher sensitivity, reduced time process, greatest biosafety and robustness.

keywords: Mycoplasma, real time PCR, SYBR Green 